\title{
An Interactive Digital Signage System Using Live-Video Superimposing and Quiz Slideshow
}

\author{
Hiroyuki Mitsuhara*
}

(Received 16 June 2012 and accepted in revised form 4 December 2012)

\begin{abstract}
Niche-Learning is a learning style using digital signage (DS) that lets students learn by viewing short digital learning materials (NL materials) on a large public display during class break times. At the author's university, a simple DS system has delivered NL materials for about 4 years, but many students do not view the NL materials. One cause of this undesirable situation may be that NL materials are one-sidedly delivered. Therefore, the author made the DS system interactive. The interactive DS system called "IVS" superimposes students standing in front of the display on quiz slideshows. In addition, the IVS enables the students to answer quiz questions in a slideshow by their simple body movement.

Keywords: digital signage, interaction, live-video superimposing, quiz slideshow, Kinect, Niche-Learning
\end{abstract}

\section{Introduction}

Recently, information delivery that targets limited numbers of people (viewers), for a specific time and place has attracted attention. Such information delivery is typified by digital signage (DS). DS delivers information locally to a public space using a public display to enhance the value of information ${ }^{(1)}$. For example, DS in a train delivers operational information (e.g., the next stop), news, advertisements, and short digital learning materials to each train car.

The author's group has focused on DS as a delivery medium for digital learning materials on a university campus and started the project called "NicheLearning"- coined term from "niche" representing the break time between lectures and "e-Learning". NicheLearning is a learning style through DS and means that students learn by viewing short digital learning materials on a large public display during their break time ${ }^{(2)}$. The short digital materials are hereafter called "NicheLearning materials" (NL materials). In this project, a simple DS system was developed and NL materials were created mainly by student volunteers ${ }^{(3)}$.

For the past 4 years in the Faculty of Engineering, at the University of Tokushima, a DS system has delivered official information, laboratory introductions, and

\footnotetext{
* Institute of Technology and Science, The University of Tokushima, Japan
}

NL materials about English conversation, disaster prevention, etc. However, it was found that many students did not view the NL materials. The author felt the major causes to be (1) the number of the NL materials was limited and (2) the NL materials were one-sidedly delivered. To treat the former cause, there is a need to bring together NL material creators (student volunteers) more actively. For the latter cause, there is a need to make the system interactive.

To motivate the students to view NL materials, first, the author attached importance to making the system interactive. Many interactive DS systems, which mainly utilize sensor devices, have been developed and used. For example, CityWall enables people to move, scale, and rotate pictures on a touch-sensitive large public display by multiple-hand-tracking functionality ${ }^{(4)}$. There are also interactive DS systems used in educational facilities. For example, Brignull et al. ${ }^{(5)}$ developed an interactive DS system where students could share and exchange multimedia data on a large public display in a high school and then reported that the system revitalized the students' social interaction through practical use. These systems do not necessarily focus on learning on a university campus. On the other hand, this study focuses on a new learning style through DS.

The remainder of this paper is organized as follows. Section 2 overviews Niche-Learning. Section 3 describes how to make Niche-Learning interactive. Section 4 briefly reports on a preliminary experiment. Section 5 summaries this study. 


\section{Niche-Learning}

Many students relax during the break time between lectures. Interpreting the latest findings in neuroinformatics $^{(6)}$ shows that human beings can learn more effectively (strengthen their memories) when they are relaxed. Therefore, break time can be regarded as a potential learning opportunity on campus even if it is short.

\subsection{Conceptual design}

The Niche-Learning project provides students with a short-time learning opportunity in public spaces on campus during the break time; in Niche-Learning, "who" views NL materials is students and "when" the students view the NL materials is the break time. Figure 1 shows the design overview of Niche-Learning.

\subsubsection{Where students view NL materials}

Students often spend their break time together with friends in a lounge area. Or, they walk out of a lecture building soon after the lecture ends. To turn the break time reasonably into a short-time learning opportunity, the DS system is installed in the lounge area and the entrance hall.

If large public displays are installed in many such public spaces, the students can view NL materials casually and frequently.

\subsubsection{What NL materials students view}

The break time is short. In many cases, it is less than 15 min except for the lunch break (about $1 \mathrm{~h}$ ). In light of this situation, NL materials should be concise. In addition, video and animations are more attractive than text information and still images. Therefore, short videos and slideshows are adopted as the main NL materials, each of which is less than $15 \mathrm{~min}$.

If many NL materials are delivered, the students can acquire wide knowledge in various fields.

\subsubsection{How students view NL materials}

In Niche-Learning, students happen to view NL materials together with friends and students whom they do not know. In other words, Niche-Learning is de-

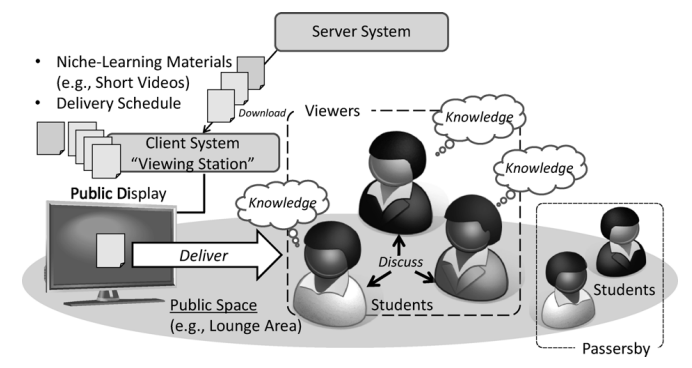

Figure 1. Design Overview of Niche-Learning.

signed for many unspecified students who may not have certain purposes; in Niche-Learning, "why" the students view the NL materials will depend on various conditions (e.g., students, time, and place).

If many unspecified students have an interest in the same NL material in the same space, they can discuss it together and learn about it beyond what appears as the DS content.

\subsection{System}

The DS system for Niche-Learning has a simple composition and allows an administrator to select one of the following operation modes: "network" and "standalone". The stand-alone mode is needed because the computer network is not always available on campus. When the network mode is selected, a client system called a "Viewing Station" (VS) communicates with an external server system (physically-separated).

\subsubsection{Client system}

The VS, working on the Microsoft .NET framework, is connected with a large public display and controls NL material delivery according to a delivery schedule. In the network mode, the VS automatically downloads NL material files (e.g., MPEG and PPT/PPS) and a delivery schedule file (XML) from the server system to an internal specified directory. In the stand-alone mode, the administrator manually uploads those files to the directory via a USB flash drive.

When a delivery time comes, the VS activates Windows Media Player or PowerPoint and opens the corresponding NL material file on the activated software. 


\subsubsection{Server system}

The server system, implemented in the LAMP software combination and available in the network mode, stores and manages the NL material files and the delivery schedule file.

When receiving the request to download new NL materials and the updated delivery schedule, the server system transfers the corresponding files to the VS.

\section{Interactive Niche-Learning}

Several times the author directly observed how many students viewed NL materials in a lounge area and an entrance hall. Unfortunately, many students did not view them. The Niche-Learning was not attractive to the students. One major cause of this undesirable situation was that NL materials were one-sidedly delivered. In other words, the VS did not motivate the students to view NL materials due to no interactivity.

Therefore, the author decided to realize Interactive Niche-Learning, that is, an Interactive VS (IVS).

\subsection{Research question}

The IVS aims at attracting people being somewhat away from the display, but has to take account of suitable attracting methods for the campus environment. It may be easy to attract students by sound effects that are suddenly performed; and louder sounds will attract students from a wider area around the display. However, this method should be avoided as much as possible on campus. This is because sound effects are occasionally dissonant and may disturb relaxation on campus. Therefore, the IVS should have interactivity that directs the students' attentions to the display without sound effects.

Even if the students direct their attention to the display, if it is only for a moment, they are not provided with any learning opportunity. Therefore, the IVS should have interactivity that keeps their attention on the display, that is, it gets them interested in the delivered NL materials.

By having the two above-mentioned interactivities, the IVS can motivate the students to view NL materials. Therefore, the research question of this study was what interactivity that was not a sound effect should be imple- mented in the IVS to direct and keep students' attention on the display.

\subsection{Fundamental ideas}

The main NL materials are videos and slideshows. It is easy for the IVS to control the slideshow because a slideshow file is divided into indexed parts (slides). Therefore, the author adopted PPT slideshow for the IVS.

\subsubsection{How to direct students' attentions to display}

To direct students' attentions to a display, the IVS superimposes the students standing in front of the display on slideshow. This visual effect, which makes the students feel as if they are inside the slideshow, surprises them for a second. The author thought that this visual effect was effective primarily for a few students and the presence of a few students could turn the public space into an unusual space (atmosphere) that could direct other students' attention to the display.

There are interactive DS systems that superimpose viewers on the contents of advertisements and entertainment. Although not a DS system, a teleteaching system named "HyperMirror" uses live-video superimposing technology to prompt communication and understanding through smooth interactions between students in remote places $^{(7)}$. This system can extract only students from two live videos and superimpose the extracted students on a common backdrop. Such superimposing technology will impact students positively in terms of visual effects and motivate them to learn by unusual experiences.

\subsubsection{How to keep students' attentions to display}

The students who are surprised by the unusual slideshow will take an interest in it and keep their attention on the display for a while. However, if it is not reasonable to superimpose the students on the slideshow, they will lose interest in the display.

To keep the students' attention on the display for a longer time, therefore, the IVS enables them to answer quiz questions in the slideshow using a contactless sensor. Furthermore, the IVS can change the next slide depending on their answer. This interactivity leads to adaptive slideshow delivery for an increasing learning effect. For example, when answering a quiz question correctly, 
the students will be given the next slide about an advanced topic. When answering incorrectly, the students will be given the next slide about a fundamental topic.

The IVS adopts Kinect, a contactless multiple sensor device (including an RGB camera, depth sensor, and multi-array microphone) because a touch-sensitive display may not necessarily be installed within reaching distance on campus. Kinect allows students to answer the quiz questions by their body movements.

\subsection{System implementation}

\subsubsection{System composition}

The IVS is implemented on the basis of the VS. Figure 2 shows the system composition of the IVS. Kinect is set up above or below the public display. The IVS captures the video (consecutive images) shooting the area in front of the display through the Kinect.

\subsubsection{Configurations}

For the IVS, the following configurations for each slide in a slideshow (PPT file) are added to the delivery

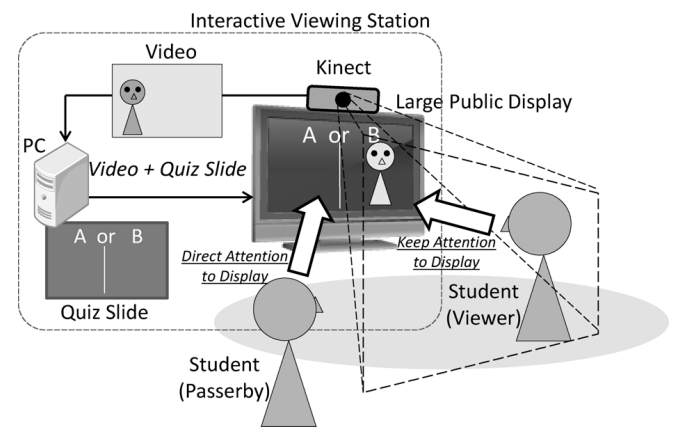

Figure 2. System Composition of IVS. schedule (XML file):

(a) How many seconds this slide is displayed for.

(b) Which slide is the next one.

(c) Whether the captured video is superimposed on this slide.

(d) Which superimposing method is applied.

(e) Whether this slide shows a quiz question.

(f) How many options $(2,3$, or 4$)$ the quiz question has.

(g) Which option is the correct answer.

(h) Which slide is the next one when correct.

(i) Which slide is the next one when incorrect.

Every slide has a unique number. The IVS displays the slides according to the configurations of (a) and (b). A slideshow creator (e.g., teacher) has to prepare quiz slides in accordance with the fixed formats depending on the number of options.

\subsubsection{Superimposing methods}

Various superimposing methods should be implemented to direct students' attention to the display. The IVS has the following three superimposing methods. In all the methods, first of all, the captured video is mirrorreversed by the RotateFlip method in Microsoft .NET framework (.NET FW).

(1) Student Extraction Superimposing (SES)

The processing steps in this method are as follows:

i. Human bodies (students) are extracted from the mirror-reversed video by the $\mathrm{xn}:$ :UserGenerator class in OpenNI library.

ii. The two-dimensional area outside the extracted students is made transparent by the TransparencyKey property in .NET FW.

iii. The extracted students are superimposed on an anterior layer of the slide.

Figure 3(a) shows an example implementation of this method. In Figure 3(a), the extracted student blocks out the characters on the slide.

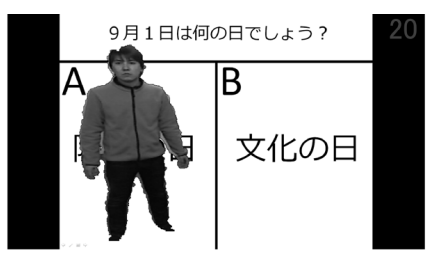

(a) Student Extraction Superimposing (SES)

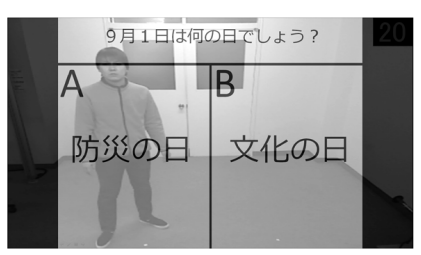

(b) Sub-transparent Superimposing (STS)

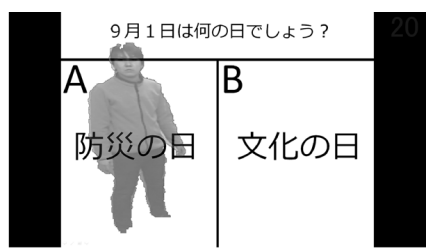

(c) Combined Superimposing (SES+STS)

Figure 3. Implemented Superimposing Method. 
(2) Sub-transparent Superimposing (STS)

The mirror-reversed video is made sub-transparent by the opacity property in .NET FW and superimposed on an anterior layer of the slide. Figure 3(b) shows an example of this method.

(3) Combined Superimposing (SES + STS)

This method combines SES and STS. The extracted students are made sub-transparent and superimposed on an anterior layer of the slide. Figure 3(c) shows an example of this method.

\subsubsection{Answering method}

The superimposed students can answer the quiz questions by making simple body movements in vertical and horizontal directions. When choosing an option of a quiz, a student moves to the corresponding option area designated (displayed) in the quiz slide and turns his/her face toward the display (Kinect).

The processing steps of this answering method are as follows:

i. Students' face areas are detected from the captured video by the HaarDetectObjects method in OpenCV library.

ii. The centroid positions of the detected face areas are calculated.

iii. For each of the centroid positions, which option area it belongs to is judged from the rectangle $(x, y)$ coordinates depending on the configuration of (f) (how many options the quiz question has).

iv. For each of the option areas, the numbers of students who currently choose it are updated. [Return to step i during answering time]

v. After the designated number of seconds has passed, the option that more of the students currently choose is determined as their final answer. If the same number of students chooses the options equally, the option of the correct answer is automatically determined as their final answer.

vi. Whether their final answer is correct or incorrect is judged from the configuration of (g) (which option is the correct answer).

\subsubsection{Slideshow controlling method}

The IVS displays the next slide depending on the students' final answer and the configurations of (h) (which slide is next when the answer is correct) and (i)

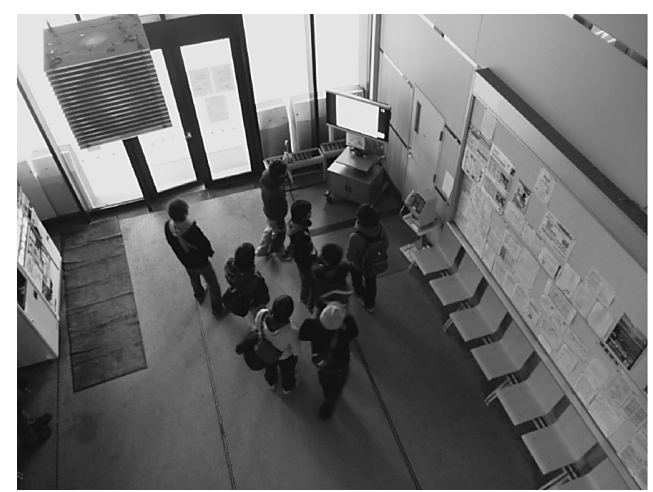

Figure 4. Students Viewing the IVS Installed in Entrance Hall for the Experiment.

(which slide is next when the answer is incorrect).

\section{Preliminary Experiment}

Focusing on the superimposing methods, a preliminary experiment was conducted in a real setting to determine how many students directed their attention to a display.

\subsection{Setting}

\subsubsection{IVS installation}

An IVS with a 40-inch LCD was installed in the busiest entrance hall of a lecture building in the Faculty of Engineering at the University of Tokushima (Figure 4). Kinect was connected with the IVS and shot the area in front of the display.

\subsubsection{Experimental period}

The experimental period was the lunch break on 16 days (Tuesday, Wednesday, Thursday, and Friday) for 4 weeks (between January and February in 2012). In particular, the author focused on the 10 minutes between 11:55 and 12:05, when many students would pass through the hall to have lunch after the lectures.

\subsubsection{Quiz slideshow}

For this experiment, eight quiz slideshows about English grammar (EG) and earthquake disaster preven- 
tion (EDP) were used because these learning topics would be interesting for the students. Each slideshow consisted of 5 quizzes with two options. Each quiz consisted of a quiz slide (a quiz question), two answer slides (corresponding to the correct answer and the incorrect answer), and two illustration slides (corresponding to the two answer slides). Each slide was displayed for 20 seconds and each slideshow was displayed for $5 \mathrm{~min}$.

\subsubsection{Delivery schedule}

In each week, the two different quiz slideshows (EG and EDP) were delivered between 11:55 and 12:05. The superimposing methods were applied only to the quiz slides. The delivery schedule, which was based on the assumption that the same students would pass through the same entrance hall just around the same time on the same day of the week, was considered to eliminate a bias of the students in the superimposing methods.

\subsubsection{How to count students}

For each slideshow delivery, the captured video from the Kinect was saved as a new image file per second during the whole delivery time (5 $\mathrm{min})$. In the saved images, the students (passersby and viewers) were cumulatively counted by sight. In each image, the passersby were counted if their whole bodies were in the image. The viewers were counted if they faced front (toward the display) even for one second.

Although the superimposing methods were applied only to the quiz slides, the students were counted in all the slides. This was because the presence of the viewers could direct other students' attention to the display after the quiz slides.

\subsection{Results and considerations}

Table 1 shows the cumulative numbers of the passersby and viewers (for $40 \mathrm{~min}$ in 4 days) and the rate of the viewers in each superimposing method. The highest rate was 0.061 (in SES+STS) and the lowest rate was 0.035 (in N/A). The rates in SES, STS, and SES + STS exceeded that in N/A. Although the numbers of the passersby in N/A and STS were almost the same, the number of the viewers in STS was 1.5 times more than that of N/A.
Table 1. Passersby and Viewers

\begin{tabular}{|l|c|c|c|}
\hline \multirow{2}{*}{$\begin{array}{c}\text { Superimposing } \\
\text { method }\end{array}$} & \multicolumn{2}{|c|}{$\begin{array}{c}\text { Cumulative number } \\
\text { (for 40 min in 4 days) }\end{array}$} & \multirow{2}{*}{$\begin{array}{c}\text { Rate } \\
\text { (V/P) }\end{array}$} \\
\hline N/A & Passersby (P) & Viewers (V) & \\
\hline SES & 7338 & 263 & 0.035 \\
\hline STS & 78240 & 604 & 0.045 \\
\hline SES+STS & 10437 & 416 & 0.053 \\
\hline
\end{tabular}

"N/A" means the usual slideshow (no superimposing).

Focusing on the rate of the viewers, the author thought that all the superimposing methods were more effective than the usual slideshow in directing the students' attention to the display. In addition, it might be notable that the rates of the superimposing methods increased about $2 \%$ more than that of N/A regardless of time when students would be busy going to lunch.

For better reliability in this experiment, the students should have been identified individually and counted only in quiz slides. However, the author considered that the potential of the implemented superimposing methods was shown through comparison in the unified counting method, including the indirect effect by the presence of the viewers.

\section{Conclusion}

This paper described the background to the need for making Niche-Learning interactive and how to realize Interactive Niche-Learning. Then the preliminary experiment focusing on whether the superimposing methods were effective in directing students' attention to the display was presented.

In addition to the preliminary experiment shown in Section 4, the author used a questionnaire in the experimental setting to determine the rate at which students kept their attention to the display for the different superimposing methods (including N/A). The questionnaire results indicated that SES, STS, and SES+STS were effective in keeping attention on the display and SES was most effective. The author thought that the students in SES could not help keeping their attention on the display to understand the quiz questions that were blocked out by their superimposed images. At this point, therefore, the author has concluded that STS and SES+STS are most effective in directing and keeping viewer atten- 
tion on the display.

From this study, many future tasks have been identified. One of them is to carry out further experiments in real settings to clarify not only the suitability of the superimposing methods and the answering method to the quiz slides but also the learning effect of Interactive Niche-Learning.

\section{Acknowledgements}

This study was supported in part by the Grant-in-Aid for Young Scientists (A) No. 24680079 from the Japan Society for the Promotion of Science. Thanks are expressed to Mr. Hidetaka Ishikubo for his efforts in implementing the IVS and conducting the experiments.

\section{References}

(1) Byrne, D., Freyne, J., Smyth, B. et al.: "Classifying public display systems: an input/output channel perspective", Proc. of Designing and Evaluating Mobile Phone-Based Interaction with Public Displays (CHI 2008 Workshop) (2008).

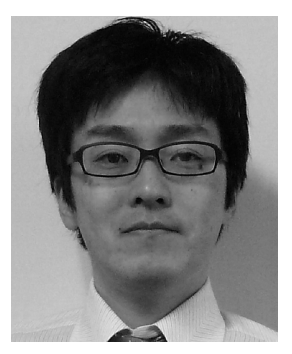

Hiroyuki Mitsuhara received the B.E. and M.E. degrees from Kinki University in 1998 and 2000, and then he received the Ph.D. degree from The University of Tokushima in 2003. He is currently an associate professor at The University of Tokushima. His research interests include entertainment computing, human-computer interaction, eLearning, and Web-based learning. Recently, he received the IEEE Young Researcher Award 2011 from IEEE Education Society Japan Chapter. He is a member of JSISE, JSET, IPSJ, IEICE, and IEEE.
(2) Mitsuhara, H., Matsumoto, J., Uosaki, N. et al.: "NicheLearning: New Learning Style Using Public Display System", Proc. of ED-MEDIA 2009, pp. 1167-1175 (2009).

(3) Matsumoto, J., Mitsuhara, H., Uosaki, N. et al.: "Learning Material Creation by Student Participation for NicheLearning", IADIS International J. on WWW/Internet, Vol. 7, No. 2, pp. 31-46 (2009).

(4) Peltonen, P., Salovaara, A., Jacucci, G. et al.: "Extending Large-Scale Event Participation with User-Created Mobile Media on a Public Display", Proc. of MUM 2007, pp. 131-138 (2007).

(5) Brignull, H. and Rogers, Y.: "Enticing People to Interact with Large Public Displays in Public Spaces", Proc. of INTERACT'03, pp. 17-24 (2003).

(6) Rutishauser, U., Ross, I.B., Mamelak, A.M. et al.: "Human memory strength is predicted by theta-frequency phase-locking of single neurons", Nature, Vol. 464, No. 7290, pp. 903-907 (2010).

(7) Nakazawa, A., Okubayashi, T., Zaorski, S. et al.: "Use of Multiple Tools in Distance Learning: International Distance Learning Using “HyperMirror"”, Proc. of APRU 9th Distance Learning and the Internet Conference 2008, pp. 119-123 (2008). 